\title{
Détection d'événements indésirables par la méthode des trigger tools à partir des dossiers de régulation au sein d'un CRRA 15
}

\section{Detection of Adverse Events by the Trigger Tools Method from Regulatory Files within a French Prehospital Medicalized Emergency Service}

\author{
M. Bechu · I. Payet - V. Bounes \\ Reçu le 12 juillet 2017; accepté le 20 janvier 2018 \\ (C) SFMU et Lavoisier SAS 2018
}

Résumé Objectif: La méthode dite des trigger tools permet une analyse objective, rapide et fiable des risques portant sur l'examen de dossiers de patients ciblés afin de mettre en évidence des d'événements indésirables (EI) évitables ou porteurs de risques (EPR). L'intérêt de cette méthode n'a jamais été étudié dans le cadre d'un centre de réception et de régulation des appels 15 (CRRA 15). L'objectif était d'évaluer la pertinence de triggers choisis au sein d'un CRRA 15 afin de mettre en place un système de détection d'EI par la méthode des trigger tools.

Matériel et méthodes : Au sein du SAMU 31, au CHU de Toulouse, les dossiers de régulation (DR) étaient extraits en cas de déclenchement de triggers prédéfinis : délais de départ SMUR, délais d'arrivée, temps de prise en charge sur place, délais de décision d'envoi d'une équipe SMUR après le premier appel, patients décédés et réorientations des patients dans les 48 heures. Le critère de jugement principal était le pourcentage d'EI évitables et d'EPR, parmi les patients dont le dossier a déclenché un trigger donné, c'est-à-dire la prévalence du trigger. Les critères de jugement secondaires étaient la gravité de chaque EI évitable selon l'AHRQ severity scale et les facteurs favorisant leur survenue.

Résultats : Du 9 juillet 2011 au 13 avril 2012, 174536 DR ont été traités, 4404 (2,5\%) ont déclenché un trigger (soit 2882 patients). On retrouvait 203 DR avec un EI, qui concernaient 153 patients ; 73 présentaient un EI évitable, 16 un EPR, 30 un EI non évitable. La prévalence de chaque trigger était de $17,6 \%$ (IC $95 \%$ : 4,8-20,4\%) pour celui

\footnotetext{
M. Bechu $(\bowtie) \cdot$ I. Payet $\cdot$ V. Bounes

Service d'aide médicale urgente de la Haute-Garonne (SAMU 31),

centre hospitalier universitaire, place du Docteur-Baylac, TSA 40031, F-31059 Toulouse, France

e-mail : bechu.m@chu-toulouse.fr

V. Bounes

Université de Toulouse, Inserm UMR 1027, 31000 Toulouse, France
}

« délai de départ », 6,7\% (IC 95\%: 3,7-9,7\%) pour le trigger " réorientation précoce », 6,4\% (IC $95 \%: 4,6$ $8,2 \%$ ) pour celui « délai de décision SMUR », 3,4 \% (IC $95 \%: 0,1-7,2 \%$ ) pour le trigger « temps sur place », $2,9 \%$ (IC $95 \%: 0,1-8,5 \%$ ) pour celui « délai d'arrivée » et $3 \%$ (IC $95 \%: 1,5-4,5 \%$ ) pour le trigger « décès ».

Conclusion: Les triggers qui semblaient les plus pertinents étaient donc " délai de décision SMUR », " réorientation précoce » et « décès ». La méthode des trigger tools permet une analyse rapide et efficace des DR, essentielle pour réduire le risque et améliorer la qualité de prise en charge des patients.

Mots clés Qualité · Préhospitalier · Événement indésirable - Trigger tools

Abstract Aims: The "trigger tools" method is an objective risk analysis for examining targeted patient records in order to identify avoidable adverse events (AE). Our objective was to evaluate the relevance of selected triggers in a French prehospital medicalized emergency service in order to implement a routine system for the detection of adverse events.

Procedure: In Toulouse University Hospital SAMU, regulatory files were automatically extracted from the patients charts based on predefined triggers: times of departure for the team, arrival time on site, assessment time, time taken by the dispatcher after the first call to send a medicalized team, deceased patients and reorientation of patients to another institution within 48 hours. The main judgment criterion was, for each trigger, the percentage of avoidable AEs, and the prevalence. Secondary judgement criteria were the severity of each avoidable AE according to the AHRQ severity scale and the factors favouring their occurrence.

Results: From July 9, 2011, to April 13, 2012, 174,536 DR were implemented, of which 4404 (2.5\%) were triggered. There were found to be 203 DR with an AE (153 patients), 73 had an avoidable AE, 16 a near miss, 30 an unavoidable 
AE. The prevalence was $17.6 \%$ (CI 95\%, 4.8\%-20.4\%) for the trigger "departure time", $6.7 \%$ (CI 95\%, 3.7\%-9.7\%) for the "early reorientation", $6.4 \%$ (CI 95\%, 4.6\%-8.2\%) for the "SMUR decision time", 3.4\% (CI 95\%, 0.1\%-7.2\%) for the trigger "time at location", $2.9 \%$ (CI 95\%, 0.1\%-8.5\%) for "time of arrival" and 3\% (CI 95\%, 1.5\%-4.5\%) for the trigger "death". Of the 11 most serious avoidable AE, 7 were linked to a "SMUR decision time" and 4 to a "death" trigger; the less serious avoidable AE were linked to "departure time" trigger.

Conclusion: The most interesting triggers were "SMUR decision time", "early reorientation" and "death". The trigger tool method enables rapid and effective analysis of regulatory files, which is essential to reduce the risk and improve the quality of patient care.

Keywords Quality · Prehospital · Adverse event · Trigger tool

\section{Introduction}

Le domaine de l'urgence, et plus particulièrement celui de la régulation médicale, est connu pour être à haut risque d'erreur et pour pouvoir générer des événements indésirables (EI). Un EI associé aux soins est un événement lié aux soins et non à l'évolution normale de la maladie, et qui aurait pu entraîner (événements porteurs de risque [EPR]) ou a entraîné un préjudice pour le patient (EI évitables) [1]. Il existe plusieurs types d'EI définis selon la Haute Autorité de santé (HAS) en fonction de leur gravité. Différentes méthodes existent afin de les détecter. La méthode dite des trigger tools (ou événements déclencheurs d'analyse de risque selon la HAS) a été introduite par Jick pour la première fois en 1974 [2,3]. Elle a été secondairement redéfinie et modernisée par l'utilisation de triggers électroniques intégrés à un système d'information hospitalier permettant d'identifier des dossiers à analyser à la recherche d'un EI [4,5]. Cette méthode consiste à analyser des dossiers sélectionnés dont la détection de départ porte sur la recherche d'une liste de valeurs anormales ou de situations définies par l'équipe médicale (les « triggers ») que l'on pense pouvoir être en relation avec l'occurrence d'un EI. Une fois les dossiers à risque isolés, une analyse plus fine permet de détecter les EI. La méthode des trigger tools n'a jamais été testée dans le cadre d'un centre de réception et de régulation des appels 15 (CRRA 15).

Notre travail avait pour but d'évaluer la pertinence de triggers choisis au sein d'un CRRA 15, afin de permettre de détecter les EI évitables ou porteurs de risque en fonction de l'occurrence de chaque trigger dans les DR.

\section{Matériel et méthodes}

Nous avons réalisé une étude monocentrique d'analyse qualitative au CRRA 15 de Toulouse se déroulant a posteriori sur une période de neuf mois, du 9 juillet 2011 au 13 avril 2012. L'objectif principal était de déterminer la pertinence des triggers, c'est-à-dire de calculer pour chaque trigger mis en place le pourcentage d'EI évitables ou porteur de risque parmi les patients dont le dossier présente un des triggers de la liste prédéterminée. Les objectifs secondaires étaient d'évaluer la gravité des EI évitables selon l'Agency for Health Research and Quality (AHRQ) Severity Scale [6], ainsi que de décrire leurs facteurs favorisants. L'échelle utilisée de 0 à 6 est destinée à mesurer l'impact du dommage sur la capacité fonctionnelle du patient, incluant sa qualité de vie :

- 6 : décès $\rightarrow$ décès lors de la prise en charge ;

- 5 : préjudice permanent sévère $\rightarrow$ lésions physiques ou psychologiques graves ou défigurations qui interfèrent de manière significative avec la qualité fonctionnelle ou la qualité de vie ;

- 4 : préjudice permanent modéré $\rightarrow$ lésions physiques ou psychologiques qui interfèrent de manière significative avec la qualité fonctionnelle ou la qualité de vie ;

- 3 : préjudice minime $\rightarrow$ lésions physiques ou psychologiques, mais probablement pas permanentes ;

- 2 : traitement additionnel $\rightarrow$ lésion limitée à une intervention surajoutée lors de l'hospitalisation et/ou à un allongement de la durée de séjour sans autre dommage ;

- $1:$ inconfort $\rightarrow$ inconfort, mais sans nécessité de traitement supplémentaire ni autre conséquence pour le patient ;

- 0 : pas de préjudice $\rightarrow$ aucune lésion évidente, mais des circonstances qui augmentent la probabilité d'un EI pour le patient.

Les critères d'inclusion correspondent au déclenchement d'un des triggers tels que définis ci-dessous. Ces triggers ont été choisis de façon arbitraire par des médecins du service au sein de l'UF qualité :

- trigger décès (décès d'un patient avant l'arrivée d'un équipage SMUR ou lors de la prise en charge préhospitalière) ;

- trigger délai de départ (délai entre le déclenchement d'un équipage SMUR et son départ de la base supérieur à 7 minutes) ;

- trigger délai d'arrivée (délai entre le départ de la base de l'équipage SMUR et son arrivée sur les lieux de l'intervention, supérieur à 30 minutes);

- trigger temps sur place (délai entre l'arrivée sur les lieux de l'intervention de l'équipage SMUR et son départ supérieur à 45 minutes) ; 
- trigger réorientation précoce (intervention avec transport du patient vers une structure hospitalière suivie dans les 48 heures d'une seconde intervention médicalisée ou paramédicalisée pour le même patient vers une autre structure hospitalière);

- trigger délai de décision SMUR (délai entre l'appel initial au CRRA 15 et l'envoi d'un équipage SMUR supérieur à 45 minutes).

Les critères d'exclusion étaient définis par des horaires manquants pour les triggers « délai de départ », « délai d'arrivée » et « temps sur place », ou une identité inconnue notée $\mathrm{X}$ dans le dossier de régulation pour le trigger « réorientations précoces ». Le critère de jugement principal était, pour chaque catégorie de trigger, le pourcentage d'EI évitables et EPR parmi les patients dont le dossier a déclenché un de ces trigger, c'est-à-dire la prévalence du trigger. Un trigger sera considéré comme pertinent si sa prévalence est strictement supérieure à $5 \%$. Les critères de jugement secondaires étaient la gravité de chaque EI évitable selon l'AHRQ Severity Scale et les facteurs favorisant leur survenue.

Cette étude a nécessité une analyse détaillée des DR ayant déclenché un trigger par deux médecins urgentistes du service. Lorsqu'un trigger était déclenché, les éléments suivants : date et horaires de prise en charge, lieux d'intervention et d'orientation du patient et données de l'observation médicale étaient extraits de manière automatique du logiciel de régulation médicale APPLISAMU ${ }^{\odot}$ (Appligos, Strasbourg, France) à l'aide de requêtes informatiques préétablies mises en place par un informaticien du service. Une première analyse de tous ces dossiers était effectuée par le premier médecin afin d'exclure les dossiers ne présentant pas d'EI de manière évidente. Tous ces dossiers étaient ensuite réanalysés conjointement par ces deux médecins afin de mettre en évidence les EI évitables et les EPR à l'aide d'une méthodologie inspirée de celle de l'IHI Trigger Tool.

Dans cette étude, la qualité de la prise en charge était analysée selon qu'elle correspondait ou non au standard de soins. Celui-ci correspond au degré d'attention, de précaution et de prudence qu'une personne raisonnable exercerait dans les mêmes conditions, et en tenant compte des données de la science [7]. Selon la présence ou l'absence d'un EI et/ ou selon que la prise en charge correspondait ou non au standard de soins, quatre situations étaient possibles, telles que résumées dans le tableau 1.

Les facteurs favorisant la survenue d'un EI évitable ou d'un EPR étaient recherchés et classés selon six grandes catégories définies par la HAS et utilisées dans les réunions de morbimortalité [8]. Ces facteurs favorisants pouvaient être : liés à un contexte institutionnel (contexte réglementaire, politique et social, contraintes économiques et financières, liens avec d'autres organisations ou établissements), liés à l'organisation et au management (organisation et gouvernance,

\begin{tabular}{|c|c|c|}
\hline & Standard de soins & $\begin{array}{l}\text { Pas de standard } \\
\text { de soins }\end{array}$ \\
\hline $\begin{array}{l}\text { Événement } \\
\text { indésirable }\end{array}$ & $\begin{array}{l}\text { Événement } \\
\text { indésirable non } \\
\text { évitable }\end{array}$ & $\begin{array}{l}\text { Événement } \\
\text { indésirable } \\
\text { évitable }\end{array}$ \\
\hline $\begin{array}{l}\text { Pas d'événement } \\
\text { indésirable }\end{array}$ & $\begin{array}{l}\text { Fonctionnement } \\
\text { normal }\end{array}$ & $\begin{array}{l}\text { Événement } \\
\text { porteur de risque }\end{array}$ \\
\hline
\end{tabular}

moyens affectés, niveau de décision, rapports hiérarchiques, gestion des ressources humaines, information, gestion des risques, culture qualité et sécurité), liés à l'environnement du travail (matériel, équipements, fournitures, charges de travail, conditions de travail), liés au fonctionnement de l'équipe (communication orale et écrite, supervision, mode de transmission des informations, leadership, composition, dynamique et interaction entre les personnes), liés aux procédures opérationnelles (conception, répartition, lisibilité des tâches et activités à effectuer : existence, validation, actualisation, disponibilité, faisabilité et acceptabilité des procédures opérationnelles), individuels (capacités techniques, relationnelles, compétences, formation, motivation, stress ou fatigue, état physique et psychologique, adaptations), liés au patient (antécédents, traitements, statuts, environnement social et familial, personnalité, difficulté de compréhension).

\section{Analyse statistique}

Les statistiques descriptives ont été effectuées avec le logiciel EXCEL $^{\circ}$ (Microsoft corporation, Richmond, ÉtatsUnis). Pour chaque trigger, la prévalence était calculée (nombre d'EI évitables sur nombre d'occurrences du trigger). Les résultats étaient exprimés en valeur absolue et en pourcentage.

\section{Résultats}

Sur la période du 9 juillet 2011 au 13 avril 2012, 174536 DR ont été traités. Tous les DR ont été soumis à l'analyse par la méthode des triggers. Au total, 4404 DR (2,5\%) ont déclenché un trigger, ce qui représentait 2882 patients (Fig. 1). Deux mille quatre cent soixante-deux DR ont été exclus, soit 1255 patients : 1207 en raison d'une identité notée X pour le trigger « réorientation précoce », 35 en raison de doublons pour le trigger « délai de décision SMUR », enfin 13 dossiers exclus en raison d'horaires manquants.

Le premier médecin a analysé en première intention 1942 DR qui correspondaient à 1627 patients. Parmi ces DR, 1739 ne présentaient pas d'EI de manière évidente (soit 


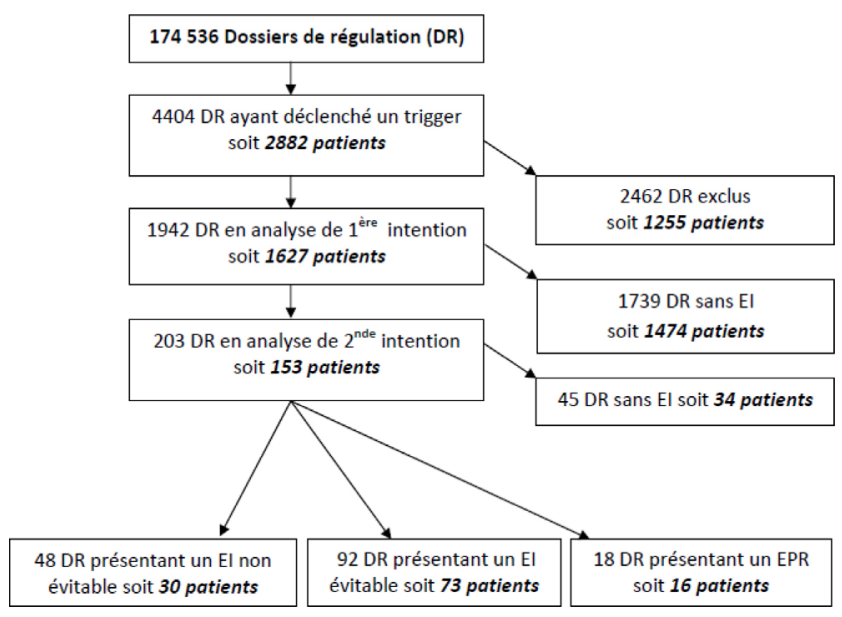

Fig. 1 Diagramme d'analyse : DR : dossier de régulation ; EI : événement indésirable; EPR : événement porteur de risque

1474 patients). Les 203 DR restants étaient soumis à l'analyse en seconde intention. Ils correspondaient à 153 patients, soit 30 ayant présenté un EI non évitable, 73 un EI évitable et 16 un EPR. Deux triggers différents ont été déclenchés pour sept patients.

La répartition des EI en fonction du type de trigger est la suivante : 44 EI évitables ou EPR étaient détectés par le trigger « délai de décision SMUR », 21 par celui « réorientation précoce », 14 par le trigger « décès », 6 par celui « délai de départ », 3 par le trigger « temps sur place » et 1 par celui " délai d'arrivée ».

Le tableau 2 présente le nombre d'EI évitables et d'EPR par type de trigger ainsi que le nombre d'occurrences de chaque trigger. La prévalence de chaque trigger était de 17,6\% (IC $95 \%: 4,8-20,4 \%$ ) pour celui « délai de départ»; $6,7 \%$ (IC $95 \%: 3,7-9,7 \%$ ) pour le trigger « réorientation précoce »; 6,4\% (IC $95 \%: 4,6-8,2 \%$ ) pour celui « délai de décision SMUR »; 3,4 \% (IC $95 \%$ : 0,1-7,2\%) pour le trigger « temps sur place »; 2,9\% (IC 95\%:0,1-8,5\%) pour celui « délai d'arrivée » et $3 \%$ (IC $95 \%: 1,5-4,5 \%$ ) pour le trigger « décès ».
La gravité des EI évitables pour chaque type de trigger, évaluée selon l'AHRQ severity scale, est représentée dans le tableau 3. Les EI évitables les plus graves classés 6 sur l'AHRQ Severity Scale étaient au nombre de 12: 7 étaient reliés au trigger « délai de décision SMUR » et 5 au trigger « décès ». Les EI évitables les plus fréquents étaient de gravité 3 selon l'AHRQ Severity Scale et étaient reliés pour 15 patients au trigger " délai de décision SMUR », pour 5 au trigger " réorientation précoce » et pour 1 au trigger « délai d'arrivée ». Pour chaque EI évitable, les facteurs favorisant sa survenue sont notés dans le tableau 4. Il pouvait exister plusieurs facteurs favorisants par EI évitable.

\section{Discussion}

Les résultats de notre étude montrent que sur $174536 \mathrm{DR}$, 92 DR présentaient un EI évitable et 18 un EPR selon la méthode des trigger tools. Tous les triggers étudiés n'avaient pas la même pertinence. En effet, les triggers « délai de départ », « délai de décision SMUR » et « réorientation précoce » permettaient de détecter le plus d'EI évitables par rapport à leur nombre d'occurrences. Cependant, en regard du peu d'occurrences du trigger " délai de départ », les triggers permettant de détecter le plus d'EI étaient les triggers " délai de décision SMUR » et "réorientation précoce». Le trigger « délai d'arrivée » n'a mis en évidence qu'un EI évitable bien que sa prévalence soit de $2,9 \%$.

$\mathrm{Au}$ total, les triggers qui semblent les plus intéressants à étudier étaient :

- les triggers « délai de décision SMUR » et « réorientation précoce » qui permettaient de mettre en avant le plus d'EI évitables, le trigger « délai de décision SMUR » permettant en outre de détecter les EI évitables les plus graves ;

- le trigger " décès » qui permettait de détecter les décès pouvant être liés à une prise en charge ne respectant pas le standard de soins ;

Tableau 2 Événements indésirables évitables, événements porteurs de risque et prévalence de chaque trigger

\begin{tabular}{|llll|}
\hline & EI évitable et EPR & $\begin{array}{l}\text { Nombre d'occurrence } \\
\text { du trigger }\end{array}$ & Prévalence (\%) et IC 95 \% \\
\hline Trigger réorientation précoce & 21 & 315 & $6,7(3,7-9,7)$ \\
Trigger décès & 14 & 472 & $3(1,5-4,5)$ \\
Trigger délai décision SMUR & 44 & 685 & $6,4(4,6-8,2)$ \\
Trigger temps sur place & 3 & 87 & $3,4(0,1-7,2)$ \\
Trigger délai de départ & 6 & 34 & $17,6(4,8-20,4)$ \\
Trigger délai d'arrivée & 1 & 34 & $2,9(0,1-8,5)$ \\
\hline Les données sont exprimées en nombre et en pourcentage : EI : événement indésirable ; EPR : événement porteur de risque ; IC \\
$95 \%$ : intervalle de confiance à 95 \%
\end{tabular}


Tableau 3 Gravité des événements indésirables évitables et des événements porteurs de risque selon le type de trigger

\begin{tabular}{|c|c|c|c|c|c|c|c|c|}
\hline & \multicolumn{8}{|c|}{ AHRQ Severity Scale } \\
\hline & $\mathbf{0}$ & 1 & 2 & 3 & 4 & 5 & 6 & Total \\
\hline Trigger réorientation précoce & 2 & 0 & 5 & 5 & 7 & 2 & 0 & 21 \\
\hline Trigger décès & 9 & 0 & 0 & 0 & 0 & 0 & 5 & 14 \\
\hline Trigger délai décision SMUR & 3 & 0 & 10 & 15 & 5 & 4 & 7 & 44 \\
\hline Trigger temps sur place & 0 & 0 & 0 & 1 & 1 & 1 & 0 & 3 \\
\hline Trigger délai de départ & 2 & 2 & 2 & 0 & 0 & 0 & 0 & 6 \\
\hline Trigger délai d'arrivée & 0 & 0 & 1 & 0 & 0 & 0 & 0 & 1 \\
\hline Total & 16 & 2 & 18 & 21 & 13 & 7 & 12 & 89 \\
\hline
\end{tabular}

Tableau 4 Facteurs favorisant les événements indésirables selon la Haute Autorité de santé [8]

\begin{tabular}{|ll|}
\hline & Événements indésirables \\
\hline Contexte institutionnel & 0 \\
Organisation et management & 0 \\
Environnement de travail & 0 \\
Fonctionnement de l'équipe & 13 \\
Procédures opérationnelles & 22 \\
Individuels & 29 \\
Patient & 0 \\
\hline
\end{tabular}

- le trigger « délai de départ » dont la prévalence était la plus importante, bien que détectant peu d'EI, ces derniers ayant un score de gravité bas.

L'analyse des triggers « temps sur place » et « délai d'arrivée » ne permettait de mettre en évidence que très peu d'EI évitables. La poursuite de leur analyse ne semble donc pas pertinente. Notre étude confirmait l'idée que, pour chaque EI, les facteurs favorisants étaient multiples. C'est en effet l'association de plusieurs facteurs plus ou moins intriqués qui aboutissait à un EI. Cette méthode d'analyse est en développement, car elle ouvre la perspective d'une méthode rapide et fiable portant sur l'examen des dossiers médicaux des patients. En effet, la déclaration spontanée des EI par les professionnels de santé a montré ses limites : sous déclaration, biais dans la déclaration et aspect chronophage de cette méthode [9]. L'analyse des dossiers par la méthode des triggers s'affranchit donc de la difficulté de déclaration par le personnel soignant.

Dans notre étude, la liste des triggers utilisés avait été déterminée par les membres de l'UF « démarche qualité en médecine d'urgence préhospitalière » en raison de l'absence de triggers dans le domaine particulier de la régulation médicale, dont nous avons validé la pertinence pour certains. Il existe, en revanche, des listes de triggers définis pour les patients en contexte hospitalier, par exemple par l'IHI Trigger Tool ou par la HAS. Dans les études déjà réalisées, les dossiers étaient soit tirés au sort puis analysés manuellement à la recherche de triggers prédéfinis [10] que l'on pensait à risque d'être reliés à un EI, soit analysés de manière informatisée et systématisée $[4,11]$ comme dans notre étude. Cette dernière méthode permettait d'avoir une analyse exhaustive, tous les dossiers étant soumis au screening des triggers. Les triggers ne permettent alors que de signaler les dossiers à analyser, la seconde analyse étant dans tous les cas manuelle. L'utilisation des triggers a été jusque-là testée et enseignée dans des domaines variés tels que les EI liés aux médicaments aussi bien chez les patients ambulatoires $[3,5,12]$ qu'hospitalisés $[11,13,14]$, les EI liés à une hypoglycémie chez l'enfant [15] et ceux dans une unité de néonatologie [16]. Mais ils ont également été étudiés de manière globale [12], par exemple avec l'Institute for Healthcare Improvement (IHI) Global Trigger Tool. Il s'agit d'un outil permettant une analyse manuelle rétrospective d'un échantillon de dossiers de patients hospitalisés, sélectionné au hasard, à la recherche de triggers positifs et d'événements en rapport $[17,18]$. Dans le cadre de la médecine d'urgence, l'intérêt des triggers a également été démontré au service d'accueil des urgences (SAU). Une étude monocentrique taïwanaise a montré que l'utilisation des triggers permet de détecter plus d'EI évitables que d'autres méthodes de signalement d'EI dans un SAU [19]. Une étude récente [20] a identifié un certain nombre de triggers applicables à la médecine d'urgence à l'aide de la littérature. Ils ont étudié neuf indicateurs d'EI dans deux SAU au Qatar et aux États-Unis. Divers triggers cliniques, comme celui de la baisse de la PA de plus de $20 \%$ par rapport à la PA initiale, se sont avérés très pertinents. Le délai entre deux décisions médicales ou entre deux consultations dans un SAU semble être un indicateur fiable dans la détection des EI. Au niveau international, les études montrent un taux de consultations dans les 72 heures suivant une admission dans un SAU de 0,5 à 
$3 \%$ [21] aux États-Unis et jusqu'à $10 \%$ [22] pour les patients signant une décharge de soins. Ce taux était de $2,2 \%$ à Singapour [23]. Un travail réalisé dans deux SAU [24] canadiens en 2010 utilisant cette méthode de détection d'EI dans cette population avait trouvé une prévalence de $12 \%$ avec le trigger « nouvelle consultation dans les 72 heures suivant une admission avec nécessité d'hospitalisation ». Les trigger tools sont également utilisés dans les services d'urgences pour ce qui est de la détection des EI liés aux médicaments [25] ainsi que dans le transport héliporté de patients nécessitant une ventilation invasive [26]. Cependant, cette méthode n'a jamais été étudiée dans le cadre particulier d'un CRRA 15.

Notre étude présente toutefois des limites telles que l'existence de données manquantes dans le DR. Dans ce cas-là, ces dossiers ont été exclus de l'analyse. De plus, il s'agissait d'une analyse sur dossiers sans interrogatoire des patients ni des médecins concernés. Une part de subjectivité dans l'analyse était donc possible.

\section{Conclusion}

Parmi les six triggers préétablis, trois ont donc été retenus : " délai de décision SMUR », « réorientation précoce », et « décès » pour être étudiés de manière prospective au sein du service. La méthode des trigger tools permet ainsi une analyse rapide et efficace des DR pour mettre en évidence d'éventuels EI évitables et ainsi améliorer nos pratiques. Cette étude étant rétrospective, il pourrait être intéressant d'analyser ces dossiers de manière prospective et continue pour favoriser une attention sur la sécurité des patients et sur la démarche qualité d'un service.

Liens d'intérêts Les auteurs déclarent ne pas avoir de liens d'intérêts.

\section{Bibliographie}

1. Agence nationale d'accréditation et d'évaluation en santé (2003) Principes méthodologiques pour la gestion des risques en établissement de santé. http://nosobase.chu-lyon.fr/recommandations/ anaes/2003_qualite_methodologie_ANAES.pdf (Dernier accès le 18 janvier 2018)

2. Haute Autorité de santé (HAS) (2012) La sécurité des patients. Mettre en œuvre la gestion des risques associés aux soins en établissement de santé. Des concepts à la pratique. https://www.hassante.fr/portail/upload/docs/application/pdf/2012-04/guide_gdr_pages1a64.pdf (Dernier accès le 18 janvier 2018)

3. Jick H (1974) Drugs--remarkably nontoxic. N Engl J Med 291:824-8

4. Classen DC, Pestotnik SL, Evans RS, et al (1992) Description of a computerized adverse drug event monitor using a hospital information system. Hosp Pharm 27:774-83
5. Evans RS, Pestotnik SL, Classen DC, et al (1998) A computerassisted management program for antibiotics and other antiinfective agents. N Engl J Med 338:232-8

6. Agency for Healthcare Research and Quality (AHRQ) (2013) Common formats for patient safety organizations - Version 1.2 (2013), Users Guide. https://www.pso.ahrq.gov/common (Dernier accès le 18 janvier 2018)

7. Strauss DC, Thomas JM (2010) What does the medical profession mean by "Standard of Care?" J Clin Oncol 27:e192-e3

8. Haute Autorité de santé (HAS) (2009) Évaluation et amélioration des pratiques : revue de mortalité et de morbidité. https://www. has-sante.fr/portail/upload/docs/application/pdf/2009-08/guide_rmm_juin_09.pdf (Dernier accès le 18 janvier 2018)

9. Leape LL (1999) Why should we report adverse incidents? J Eval Clin Pract 5:1-4

10. Resar RK, Rozich JD, Simmonds T, et al (2006) A trigger tool to identify adverse events in the intensive care unit. Jt Comm J Qual Patient Saf 32:585-90

11. Szekendi MK, Sullivan C, Bobb A, et al (2006) Active surveillance using electronic triggers to detect adverse events in hospitalized patients. Qual Saf Health Care 15:184-90

12. Resar R, Rozich J, Classen D (2003) Methodology and rationale for the measurement of harm with trigger tools. Qual Saf Health Care 12:39-45

13. Classen D, Pestotnik S, Evans R, et al (2005) Computerized surveillance of adverse drug events in hospital patients. Qual Saf Health Care 14:221-6

14. Rozich J, Haraden C, Resar R (2003) Adverse drug event trigger tool: a practical methodology for measuring medication related harm. Qual Saf Health Care 12:194-200

15. Dickerman MJ, Jacobs BR, Vinodrao H, et al (2011) Recognizing hypoglycemia in children through automated adverse-event detection. Pediatrics 127:e1035-e41

16. Sharek PJ, Horbar JD, Mason W, et al (2006) Adverse events in the neonatal intensive care unit: development, testing, and findings of an NICU-focused trigger tool to identify harm in North American NICUs. Pediatrics 118:1332-40

17. Good VS, Saldaña M, Gilder R, et al (2011) Large-scale deployment of the Global Trigger Tool across a large hospital system: refinements for the characterisation of adverse events to support patient safety learning opportunities. BMJ Qual Saf 20:25-30

18. Adler L, Denham CR, McKeever M, et al (2008) Global trigger tool: implementation basics. J Patient Saf 4:245-9

19. Lee WH, Zhang E, Chiang CY, et al (2017) Comparing the outcomes of reporting and trigger tool methods to capture adverse events in the emergency department. J Patient Saf 10:1097

20. Howard IL, Bowen JM, Al Shaikh LAH, et al (2017) Development of a trigger tool to identify adverse events and harm in emergency medical services. Emerg Med J 34:391-7

21. Pierce JM, Kellerman AL, Oster C (1990) « Bounces »: an analysis of short-term return visits to a public hospital emergency department. Ann Emerg Med 19:752-7

22. Ross MA, Hemphill RR, Abramson J, et al (2010) The recidivism characteristics of an emergency department observation unit. Ann Emerg Med 56:34-41

23. Kuan WS, Mahadevan M (2009) Emergency unscheduled returns: can we do better? Singapore Med J 50:1068-71

24. Calder L, Pozgay A, Riff S, et al (2015) Adverse events in patients with return emergency department visits. BMJ Qual Saf 24:142-8

25. Karpov A, Parcero C, Mok CP, et al (2016) Performance of trigger tools in identifying adverse drug events in emergency department patients: a validation study. Br J Clin Pharmacol 82:1048-57

26. Patterson PD, Lave JR, Martin-Gill C, et al (2014) Measuring adverse events in helicopter emergency medical services: establishing content validity. Prehosp Emerg Care 18:35-45 